\title{
Desempenho e morfometria intestinal de juvenis de tilápia-do-nilo alimentados com dietas suplementadas com L-glutamina e L-glutamato
}

\author{
Lilian Carolina Rosa da Silva ${ }^{1}$, Wilson Massamitu Furuya ${ }^{2}$, Maria Raquel Marçal Natali ${ }^{3}$, \\ Christiano Rodrigues Schamber ${ }^{1}$, Lilian Dena dos Santos ${ }^{1}$, Luiz Vitor Oliveira Vidal ${ }^{1}$
}

\footnotetext{
${ }^{1}$ Programa de Pós-graduação em Zootecnia, Universidade Estadual de Maringá - UEM, Av. Colombo, 5790, Maringá - PR.

2 Departamento de Zootecnia - UEM, Maringá - PR.

${ }^{3}$ Departamento de Ciências Morfofisiológicas - UEM, Maringá - PR.
}

RESUMO - Este estudo foi realizado para avaliar níveis de L-glutamina e L-glutamato em dietas para juvenis de tilápiado-nilo $(0,60 \pm 0,1 \mathrm{~g})$. Foi utilizado delineamento inteiramente casualizado com quatro dietas e três repetições e 90 peixes por unidade experimental. Foi utilizada dieta controle, com $29 \%$ de proteína digestível e $2.940 \mathrm{kcal} / \mathrm{kg}$ de energia digestível, suplementada mistura de L-glutamina e L-glutamato na proporção de 0, 1, 2 e 3\% da dieta, durante 85 dias. Não foi observado efeito da L-glutamina e L-glutamato sobre o consumo, a conversão alimentar, a taxa de eficiência protéica, a eficiência de retenção de nitrogênio, o índice hepatossomático, a composição química corporal, a amônia e ureia sanguíneas. O aumento nos níveis de L-glutamina e L-glutamato nas dietas teve aumento linear sobre o ganho de peso e efeito quadrático na altura dos vilos. A adição de L-glutamina e L-glutamato melhora o ganho de peso e a altura das vilosidades intestinais de tilápia-do-nilo.

Palavras-chave: aminoácido, histologia, mucosa intestinal, peixe

\section{Productive performance and intestinal morphology of Nile tilapia juvenile fed diets with L-glutamine and L-glutamate}

\begin{abstract}
This work was carried out to evaluate levels of L-glutamine and L-glutamate in diets for Nile tilapia juveniles $(0.60 \pm 0.1 \mathrm{~g})$. A complete randomized experimental design with four diets and three replicates and 90 fish per experimental unit was used. It was used a control diet with $29 \%$ of digestible protein and $2,940 \mathrm{kcal} / \mathrm{kg}$ of digestible energy supplemented with L-glutamine and L-glutamate at the proportion of $0,1,2$ and $3 \%$ of diet, during 85 days. It was not observed effect of dietary L-glutamine and L-glutamate on feed intake, food conversion, protein efficiency rate, nitrogen retention efficiency, hepatic somatic index, chemical body composition, blood ammonia and urea. It was observed a linear increase on gain weight and a quadratic effet on villus height when levels of L-glutamine and L-glutamate increased. The addition of L-glutamine and L-glutamate increases the weight gain and intestinal villus height of Nile tilapia.
\end{abstract}

Key Words: amino acid, fish, histology, intestinal mucous

\section{Introdução}

A tilápia-do-nilo (Oreochromis niloticus) é uma das espécies mais indicadas para criação intensiva, pela elevada taxa de crescimento, adaptabilidade a mudanças no ambiente e habilidade no crescimento e reprodução. Além disso, apresenta facilidade de obtenção de larvas, rusticidade, possui carne com boas características organolépticas e seu filé não tem espinhas intramusculares em "Y". As exigências nutricionais de peixes variam conforme a capacidade em aproveitar o alimento fornecido e além disso, os peixes não possuem exigência verdadeira em proteína e sim de adequado balanceamento entre aminoácidos essenciais e não essenciais (Sampaio et al., 2009).

A glutamina e o glutamato são aminoácidos abundantes na circulação e no espaço intracelular e são precursores da síntese de aminoácidos, nucleotídeos, proteínas e outras moléculas biologicamente importantes (Smith, 1990). O glutamato e a glutamina possuem via metabólica comum no enterócito, pois a glutamina é metabolizada em glutamato mais amônia pela glutaminase e o glutamato também pode se transformar em glutamina pela ação da glutamina sintetase (Maiorka et al., 2000). Dessa forma, o glutamato pode substituir a glutamina em diversos de seus papéis metabólicos, 
como geração de energia e síntese de aminoácidos (Wu et al., 1995).

A suplementação de L-glutamina na dieta de suínos e aves tem sido utilizada com resultados positivos em seu desempenho, prevenindo a atrofia das vilosidades do jejuno de leitões que receberam dietas com $1 \%$ de L-glutamina (Wu et al., 1996), melhorando a eficiência alimentar (Kitt et al., 2001), o ganho de peso, o desenvolvimento da mucosa intestinal de frangos de corte (Murakami et al., 2007) e a taxa de eficiência proteica e aumentando a altura das vilosidades intestinais da carpacomum (Cyprinus carpio) (Yan \& Qiu-Zhou, 2006).

O conhecimento da mucosa intestinal dos peixes é importante para fornecer informações para os estudos de nutrição, de forma a atender às exigências nutricionais para adequado desempenho e saúde dos peixes. A mucosa intestinal de peixes teleósteos tem inúmeras projeções denominadas vilos, sem criptas na base e que possuem células indiferenciadas, que sofrem sucessivas mitoses para formação das células epiteliais do vilo (Jobling, 1995).

Avaliou-se neste trabalho a utilização de L-glutamina e L-glutamato em dietas para juvenis de tilápia-do-nilo por meio do desempenho produtivo, da composição química corporal, da morfometria da mucosa intestinal e amônia e ureia sanguíneas.

\section{Material e Métodos}

O experimento foi realizado no Laboratório de Aquicultura do Núcleo de Pesquisa em Limnologia e Aquicultura (NUPELIA) da Universidade Estadual de Maringá durante 85 dias, período de janeiro a abril de 2007.

Foram utilizadas 1.080 tilápias-do-nilo da linhagem tailandesa, revertidas durante a fase larval, com peso inicial de 0,60 $\pm 0,01 \mathrm{~g}$, originadas da Piscicultura Araucária Belmonte, Rolândia, Paraná. Os peixes foram distribuídos em 12 tanques de cimento amianto com volume útil unitário de $0,8 \mathrm{~m}^{3}$ cada, totalizando 90 peixes em cada tanque, em um delineamento inteiramente casualizado com quatro dietas e três repetições.

Foi utilizado sistema de recirculação com renovação contínua de água (7 litros/minuto/tanque) e com biofiltro central. Em cada tanque foi instalado um sistema de aeração com difusores de ar acoplados a um soprador central, mantendo o oxigênio entre 4 e $6 \mathrm{mg} / \mathrm{L}$. A temperatura variou de 27 a $30^{\circ} \mathrm{C}$. Cada tanque foi recoberto com lona preta para reduzir a produção primária.

As dietas (Tabela 1) foram formuladas com base nos valores de aminoácidos digestíveis determinados para tilápiado-nilo (Furuya et al., 2001). Uma mistura de L-glutamina e L-glutamato (mínimo de 10\% de L-glutamina) foi adicionada à dieta controle na proporção de $0,1,2$ e $3 \%$ em substituição ao milho. Todos os alimentos foram moídos em moinho tipo faca e peneira de $0,5 \mathrm{~mm}$ de diâmetro. Em seguida foi pulverizada água $\left(55^{\circ} \mathrm{C}\right)$ na proporção de $12 \%$ de seu peso total. As dietas foram aglomeradas em moedor de carne e desidratadas em estufa de ventilação forçada $\left(55^{\circ} \mathrm{C}\right)$ durante 18 horas. Posteriormente, foram desintegradas, selecionando os grânulos com diâmetro de $0,8 \mathrm{~mm}$ ( $1 \underline{\text { o }}$ ao $14 \underline{\text { o }}$ dia $), 1,18 \mathrm{~mm}$ ( $15 \underline{\text { o }}$ ao $32 \underline{\text { o }}$ dia $), 2 \mathrm{~mm}$ (33ํㅡ ao $41^{\circ}$ dia) e $3 \mathrm{~mm}$ até o final do experimento.

Cada dieta foi fornecida diariamente quatro vezes/dia, às $8,12,16 \mathrm{e} 18 \mathrm{~h}$. $\mathrm{O}$ arraçoamento foi manual e até saciedade aparente, quando não foi observada regurgitação dos grânulos.

Tabela 1 - Composição da dieta controle

\begin{tabular}{|c|c|}
\hline Ingrediente & $(\%)$ \\
\hline Quirera de arroz & 16,70 \\
\hline Farelo de soja & 35,00 \\
\hline Milho & 5,00 \\
\hline Farelo de trigo & 10,00 \\
\hline Glúten de milho & 6,00 \\
\hline Farinha de peixe & 15,00 \\
\hline Fosfato bicálcico & 1,00 \\
\hline Óleo de soja & 5,00 \\
\hline Levedura desidratada & 3,00 \\
\hline Hidrolisado protéico & 2,00 \\
\hline DL-metionina & 0,18 \\
\hline L-lisina $\mathrm{HCl}$ & 0,10 \\
\hline Suplemento mineral e vitamínico ${ }^{1}$ & 0,50 \\
\hline Vitamina $\mathrm{C}^{2}$ & 0,10 \\
\hline Sal comum & 0,30 \\
\hline Antifúngico ${ }^{3}$ & 0,10 \\
\hline $\mathrm{BHT}^{4}$ & 0,02 \\
\hline \multicolumn{2}{|l|}{ Nutricional } \\
\hline Matéria seca $(\%)^{5}$ & 92,00 \\
\hline Energia digestível $(\mathrm{kcal} / \mathrm{kg})^{7}$ & 2937,16 \\
\hline Proteína bruta $(\%)^{5}$ & 32,70 \\
\hline Proteína digestível $(\%)^{5}$ & 29,43 \\
\hline Fibra bruta $(\%)^{5}$ & 3,37 \\
\hline Extrato etéreo $(\%)^{5}$ & 6,17 \\
\hline $\operatorname{Cinzas}^{5}(\%)$ & 7,56 \\
\hline Cálcio $(\%)^{5}$ & 0,93 \\
\hline Fósforo total ${ }^{5}(\%)$ & 0,93 \\
\hline Fósforo disponível $(\%)^{6}$ & 0,43 \\
\hline $\operatorname{Lisina}^{8}(\%)$ & 1,80 \\
\hline Treonina ${ }^{8}(\%)$ & 1,38 \\
\hline Metionina $+\operatorname{cistina}^{8}(\%)$ & 1,37 \\
\hline Metionina $8(\%)$ & 0,87 \\
\hline \multicolumn{2}{|c|}{ 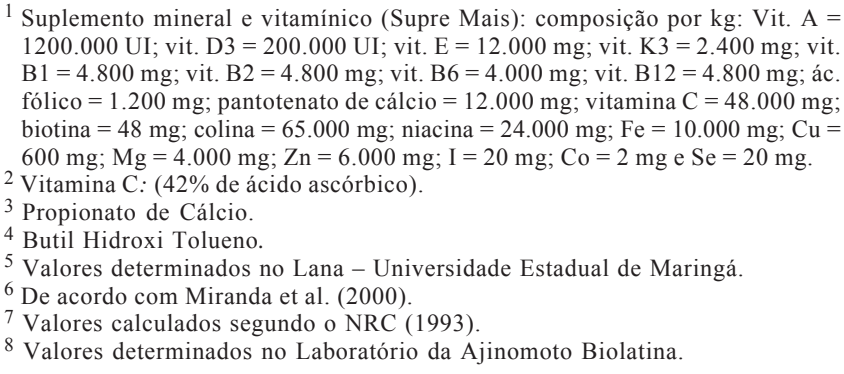 } \\
\hline
\end{tabular}


As análises químicas das dietas foram realizadas no Laboratório de Análise de Alimentos do Departamento de Zootecnia da Universidade Estadual de Maringá (LANA), segundo metodologia descrita por Silva \& Queiroz (2002). As análises de aminoácidos foram realizadas no Laboratório da Ajinomoto Biolatina pelo método de Cromatógrafo a Líquido de Alto Desempenho (HPLC), modelo Shimatzu.

Após a pesagem e distribuição dos animais em cada unidade experimental, foram coletados 30 peixes originados do mesmo lote utilizado para a montagem do experimento para determinação da composição química da carcaça inicial. Ao final do experimento, foi realizada a pesagem de todos os peixes em balança digital $(0,01 \mathrm{~g})$. Trinta peixes por repetição foram utilizados para determinação da composição química da carcaça e do índice hepatosomático, e nove peixes por dieta para a análise histológica. Os peixes foram eutanizados com superdosagem de Eugenol (300 mg/L de água).

Foram determinados o ganho de peso, o consumo da dieta e a conversão alimentar, utilizando-se para determinação do índice hepatossomático, taxa de eficiência proteica e da eficiência de retenção de nitrogênio as expressões descritas por Jauncey \& Ross (1982): IHS = $(\mathrm{PF} / \mathrm{PV}) \times 100$ em que IHS = índice hepatossomático; $\mathrm{PF}=$ peso do fígado $(\mathrm{g})$; e $\mathrm{PV}=$ peso vivo $(\mathrm{g}) ; \mathrm{TEP}=\mathrm{GP} / \mathrm{PC}$, em que TEP = taxa de eficiência protéica; $\mathrm{GP}=$ ganho de peso $(\mathrm{g})$; e PC = proteína consumida $(\mathrm{g})$; e ERN $=(\mathrm{NfX}$ Pf $-\mathrm{Ni}$ $\mathrm{XPi}) \times \mathrm{Nc}$, em que ERN = eficiência de retenção de nitrogênio; $\mathrm{Nf}=$ nitrogênio final $(\%) ; \mathrm{Pf}=$ peso final $(\mathrm{g}) ; \mathrm{Ni}=$ nitrogênio inicial $(\%) ; \mathrm{Pi}=$ peso inicial $(\mathrm{g}) ; \mathrm{e} \mathrm{Nc}=$ nitrogênio consumido $(\mathrm{g})$.

As amostras para a composição corporal dos peixes (peixe inteiro) foram moídas em moedor de carne, até obter-se uma polpa homogênea, a qual foi seca em estufa de ventilação forçada a $55^{\circ} \mathrm{C}$ por 48 horas e moídas em moinho bola. As análises químicas da carcaça foram realizadas no LANA, seguindo-se metodologia descrita por Silva \& Queiroz (2002).

A cada semana foram tomadas as medidas de temperatura ( 8 e 16 horas) e oxigênio dissolvido ( $\mathrm{mg} / \mathrm{L})$ da água de cada tanque. Os dados foram obtidos por meio de oxímetro digital portátil.

Para as análises hematológicas, foram utilizados nove peixes por tratamento. Os peixes foram anestesiados com Eugenol $(60 \mathrm{mg} / \mathrm{mL})$, o sangue foi retirado com seringa sem EDTA e colocado em tubo de ensaio com EDTA (10\%). Esses tubos foram agitados manualmente e armazenados em geladeira $\left(4^{\circ} \mathrm{C}\right)$ até posterior análise. As análises de amônia e ureia plasmática foram realizadas no Laboratório Veterinário de Análise Clínicas, Citologia e Anatomia Patológica Santo Antônio ${ }^{\circledR}$, Maringá, Paraná, utilizando-se plasma centrifugado a $2.500 \mathrm{rpm}$ por oito minutos. A análise de uréia foi realizada com o teste UV enzimático uréaseGLDH e para amônia foi utilizado o plasma com EDTA, que foi analisado pelo analisador automático Selectra XL series - da Vital Scientific.

Para a morfometria da mucosa intestinal, que foi realizada no Laboratório de Histotécnica Animal do Departamento de Ciências Morfológicas da Universidade Estadual de Maringá foram recolhidas porções de aproximadamente $5 \mathrm{~cm}$ de comprimento do intestino médio ( $45 \mathrm{~cm}$ abaixo da junção do estômago com o intestino) de 12 peixes por tratamento. As amostras foram colocadas em placa de isopor, abertas longitudinalmente, lavadas com solução salina, fixadas em solução de "Bouin" por 6 horas, desidratadas em série ascendente de álcool, diafanizadas em xilo, e incluídas em parafina, para obtenção de cortes histológicos semiseriados. Foram realizados cortes de $7 \mu \mathrm{m}$ de espessura que foram corados pelo método de hematoxilina-eosina. A fotodocumentação (captura de imagens) foi realizada no Laboratório de Captura de Imagens DCM/UEM em fotomicroscópio Olympus BX50 em objetiva de 4X, utilizando-se sistema de imagens computadorizado (Image Pro Plus - Versão 5.2-Media Cibernética). A morfometria da mucosa intestinal foi realizada em 25 vilos por animal perfazendo um total de 225 medidas por dieta, mensurando a altura dos vilos.

Os dados obtidos foram submetidos às análises de variância e regressão polinomial por meio do programa SAEG - Sistema de Análises Estatísticas e Genéticas (UFV, 1982).

\section{Resultados e Discussão}

Não foi observada mortalidade decorrente da suplementação de L-glutamina e L-glutamato durante o período experimental. Os níveis crescentes de L-glutamina e L-glutamato nas dietas não influenciaram $(\mathrm{P}>0,05)$ o consumo de dieta, a conversão alimentar, a taxa de eficiência proteína, a eficiência de retenção de nitrogênio, o índice hepatossomático e o rendimento de carcaça (Tabela 2).

Com o aumento da suplementação de L-glutamina e L-glutamato foi observado aumento linear $(\mathrm{P}<0,05)$ do ganho de peso (Tabela 2). Melhora no ganho de peso foi observada também em trabalhos com carpa-comum sob suplementação com 1,2\% de L-glutamina (Yan \& Qiu-Zhou, 2006) e leitões suplementados com $0,8 \%$ de L-glutamina na dieta (Lackeyram, 2001). O aumento no ganho de peso pode estar relacionado ao fato de a L-glutamina e o L-glutamato promoverem a síntese de aminoácidos não-essenciais para a deposição de proteína corporal. Além disso, a glutamina 
Tabela 2 - Desempenho de juvenis de tilápia-do-nilo alimentados com dietas contendo L-glutamina e L-glutamato

\begin{tabular}{|c|c|c|c|c|c|}
\hline \multirow[b]{2}{*}{ Item } & \multicolumn{5}{|c|}{ L-glutamina e L-glutamato (\%) } \\
\hline & 0 & 1 & 2 & 3 & $\mathrm{CV}(\%)$ \\
\hline Ganho de peso $^{2}(\mathrm{~g})$ & 61,22 & 62,19 & 61,58 & 66,01 & 3,21 \\
\hline Consumo de dieta (g/peixe) & 63,95 & 64,36 & 64,76 & 65,57 & 5,20 \\
\hline Conversão alimentar & 1,05 & 1,04 & 1,05 & 0,99 & 6,66 \\
\hline Taxa de eficiência protéica & 2,95 & 2,97 & 2,94 & 3,10 & 6,64 \\
\hline Eficiência de retenção de nitrogênio & 30,67 & 32,40 & 30,10 & 34,46 & 6,22 \\
\hline Índice hepato-somático & 2,29 & 2,22 & 1,98 & 2,18 & 8,03 \\
\hline
\end{tabular}

$\mathrm{CV}=$ coeficiente de variação

${ }^{2}$ Efeito linear $(\mathrm{P}<0,05)$ : Ganho de peso $\left(\mathrm{Y}=60,6902+1,3743 \mathrm{X} ; \mathrm{R}^{2}=0,75\right)$.

também pode resultar em maior ganho de peso, pelas melhorias na altura da mucosa intestinal dos animais, favorecendo a digestão e absorção de nutrientes da dieta.

Não foram observadas diferenças $(\mathrm{P}>0,05)$ na composição química corporal (Tabela 3 ) de juvenis de tilápia-do-nilo alimentados com rações contendo L-glutamina e L-glutamato. A glutamina é importante na composição do músculo esquelético, pois atua no transporte de nitrogênio entre os tecidos para a formação de aminoácidos para a síntese de proteína muscular (Forte et al., 2003). A ausência de diferenças na composição corporal dos animais pode ter ocorrido pelo fato de a glutamina exógena ter maiores efeitos em situações de estresse como infecções e queimaduras (Ribeiro et al., 2004).

A utilização de dietas com níveis crescentes de L-glutamina e L-glutamato não influenciou $(\mathrm{P}>0,05)$ os parâmetros sanguíneos de amônia e ureia (Tabela 4). Por outro lado, Flynn \& Wu (1997) suplementaram glutamina à dieta de leitões lactentes e notaram aumento da amônia sanguínea dos animais. Assim, a adição de até 3\% de L-glutamina e L-glutamato em dietas não acarreta prejuízos metabólicos em termos de formação e excreção dos resíduos nitrogenados.

As análises morfométricas das vilosidades evidenciaram efeito quadrático $(\mathrm{P}>0,05)$ da suplementação de L-glutamina e L-glutamato na altura dos vilos (Figura 1), cujo valor máximo foi de $407 \mu \mathrm{m}$ no nível de 1,67\% de L-glutamina e L-glutamato. Yan \& Qiu-Zhou (2006)

Tabela 3 - Composição química corporal de juvenis de tilápiado-nilo alimentados com dietas contendo L-glutamina e L-glutamato

\begin{tabular}{|c|c|c|c|c|c|}
\hline \multirow[b]{2}{*}{ Item } & \multicolumn{5}{|c|}{ L-glutamina e L-glutamato (\%) } \\
\hline & 0 & 1 & 2 & 3 & CV $(\%)$ \\
\hline Umidade & 72,11 & 71,61 & 72,61 & 71,45 & 1,22 \\
\hline Proteína bruta & 15,15 & 15,04 & 15,01 & 16,11 & 4,03 \\
\hline Extrato etéreo & 8,25 & 8,61 & 8,28 & 8,38 & 3,83 \\
\hline Cinzas & 3,68 & 3,68 & 3,10 & 3,51 & 8,65 \\
\hline
\end{tabular}

$\mathrm{CV}=$ coeficiente de variação.
Tabela 4 - Parâmetros sanguíneos de juvenis de tilápias-donilo alimentados com dietas contendo L-glutamina e L-glutamato

\begin{tabular}{|c|c|c|c|c|c|}
\hline \multirow[t]{2}{*}{ Item } & \multicolumn{5}{|c|}{ L-glutamina e L-glutamato (\%) } \\
\hline & 0 & 1 & 2 & 3 & $\mathrm{CV}(\%)$ \\
\hline Amônia sanguínea & 1,23 & 1,13 & 1,32 & 1,18 & 12,87 \\
\hline Ureia sanguínea & 5,00 & 4,67 & 4,67 & 5,00 & 8,37 \\
\hline
\end{tabular}

$\mathrm{CV}=$ coeficiente de variação.

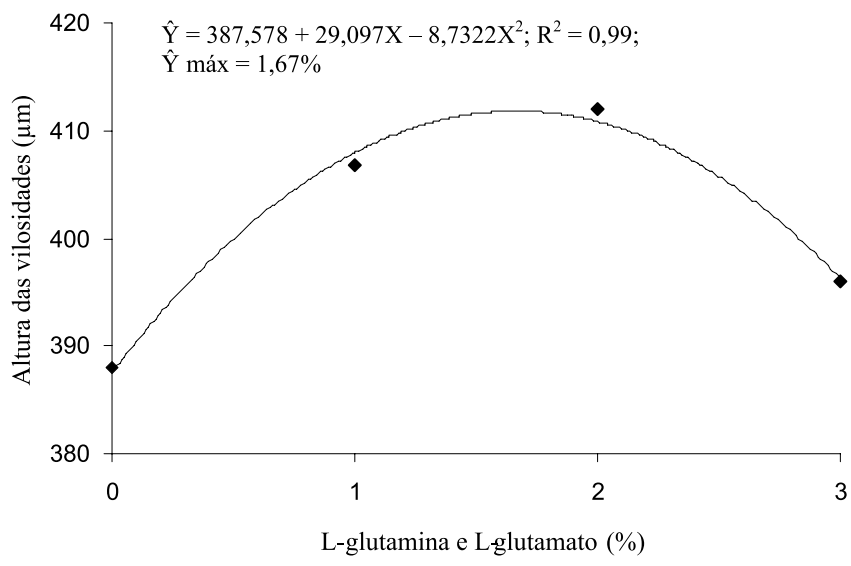

Figura 1 - Altura dos vilos intestinais de juvenis de tilápia-donilo alimentados com dietas contendo L-glutamina e L-glutamato.

também observaram aumento dos vilos intestinais com a suplementação de L-glutamina em dietas para a carpa-comum.

$\mathrm{O}$ aumento da altura dos vilos intestinais ocorreu provavelmente porque a glutamina é uma importante fonte de energia para os enterócitos, fornecendo nitrogênio para a biossíntese de nucleotídeos necessários para a replicação celular das células da mucosa do intestino (Yan \& Qiu-Zhou, 2006). Esse efeito trófico relaciona-se à renovação celular do epitélio da mucosa intestinal, pois a glutamina e o glutamato estimulam o aumento das mitoses na base do vilo, que acarreta maior proliferação 
das células do epitélio da mucosa intestinal e, dessa forma, aumentando a altura dos vilos, melhorando a digestão e absorção intestinal e o desempenho dos animais (Boleli et al., 2002).

\section{Conclusões}

A adição de L-glutamina e L-glutamato melhora o ganho de peso de juvenis de tilápia-do-nilo e, no nível de 1,67\%, é adequada para promover o desenvolvimento da mucosa intestinal.

\section{Referências}

BOLELI, I.C.; MAIORKA, A.; MACARI, M. Estrutura funcional do trato digestório. In: MACARI, M.; FURLAN, R.L.; GONZÁLES, E.P. (Eds.) Fisiologia aviária aplicada a frangos de corte. Jaboticabal: FUNEP/UNESP, 2002. p.75-96.

FLYNN, N.E.; WU, G. glucocorticoids play an important role in mediating the enhanced metabolism of arginine and glutamine in enterocytes of post weaning pigs, The Journal of Nutrition, p. 732-737, 1997.

FORTI, F.; CANCELLIERO, K.M.; SILVA, C.A. et al. O efeito da glutamina no músculo esquelético desnervado. Saúde Revista, v.5, n.9, p.59-65, 2003.

FURUYA, W.M.; PEZZATO, L.E.; MIRANDA, E.C. et al. Coeficientes de digestibilidade aparente da energia e nutrientes de alguns ingredientes pela tilápia do Nilo, Oreochromis niloticus (L.) (Linhagem Tailandesa). Acta Scientiarum, v.23, p.465-469, 2001.

GONÇALVES, G.S.; PEZZATO, L.E.; BARROS, M.M. et al. Relação lisina digestível:proteína digestível em rações para tilápias-donilo. Revista Brasileira de Zootecnia, v.38, n.12, p.22992305, 2009 .

JAUNCEY, K.; ROSS, B. A guide to tilapia feed and feeding. Scotland: University of Stirling, 1982. 111p.

JOBLING, M. Environmental biology of fishes. Fish and Fisheries Series 16. London: Chapman \& Hall. 1995. $455 \mathrm{p}$.

KITT, S.J.; MILLER, P.S.; LEWIS, A.J. et al. Effects is diet and crystalline glutamina supplementation of growth performance and small intestine morphology of weanling pigs. Journal of Animal Science, v.79, p.10, p.230-238, 2001.

LACKEYRAM, D.; YUE, X.; FAN, M.Z. Effects dietary supplementation of crystalline L-glutamine on the gastrointestinal tract and whole body growth in early-weaned piglets fed corn and soybean meal - based diets. Journal Animal Science, v.79, p.11, 2001.

MAIORKA, A., SILVA, A.V.F., SANTIN, E. et al. Influência da suplementação de glutamina sobre o desempenho e o desenvolvimento de vilos e criptas do intestino delgado de frangos. Arquivo Brasileiro de Medicina Veterinária e Zootecnia, v.52, n.5, p.487-490, 2000.

MIRANDA, E.C.; PEZZATO, A.C.; PEZZATO, L.E. et al. Disponibilidade aparente de fósforo em ingredientes pela tilápia do Nilo (Oreochromis niloticus). Acta Scientiarium, v.22, n.3, p.669-675, 2000 .

MURAKAMI, A.E.; SAKAMOTO, M.I.; NATALI, M.R.M. et al. Supplementation f glutamine and vitamin E on the morphometry of the intestinal mucosa in broiler chickens. Poultry Science, v.86, p.488-495, 2007.

NATIONAL RESEARCH COUNCIL - NRC. Nutritional requirements of fishes. Washington, D.C.: Academic Press, 1993. $114 \mathrm{p}$

RIBEIRO, S.R.; PINTO JR., P.E.; MIRANDA, A.C. et al. Weight loss and morphometric study of intestinal mucosa in rats after massive intestinal resection. influence of a glutamine-enriched diet. Revista do Hospital das Clínicas da Faculdade de Medicina de São Paulo, v.59, n.6, p.349-356, 2004.

SILVA, S.S.; QUEIROZ, S. Análises de alimentos (métodos químicos e biológicos). 2.ed. Viçosa, MG: Imprensa Universitária, 2002. 235p.

SMITH, R.J. Glutamine metabolism and its physiologic importance. Journal Parenteral and Enteral Nutrition, v.14, p.40S-44S, 1990.

UNIVERSIDADE FEDERAL DE VIÇOSA - UFV. Sistema de análises estatísticas e genéticas - SAEG. Versão 6.0. Viçosa, MG, 1982. 52p.

WU, G. Effects of concanavalin A and phorbol myristate acetate on glutamine metabolism and proliferation of porcine intraepithelial lymphocytes. Biochemistry Physiology, v. 114A, p.363-368, 1996.

WU, G.; KNABE, D.A.; YAN, W. et al. Glutamine and glucose metabolism in enterocytes of the neonatal pig. American Journal Physiology, v.137, p.R334-R342, 1995.

YAN, L.; QIU-ZHOU, X. Dietary glutamine supplementation improves structure and function of intestine of juvenile Jian carp (Cyprinus carpio var. Jian). Aquaculture, v.256, p.389-394, 2006. 